\title{
A Novel Compensation Algorithm for Thickness Measurement Immune to Lift-Off Variations Using Eddy Current Method
}

\author{
Mingyang Lu, Liyuan Yin, Anthony J. Peyton, and Wuliang Yin, Senior Member, IEEE
}

\begin{abstract}
Lift-off variation causes errors in the eddy current thickness measurements of metallic plates. In this paper, we have developed an algorithm that can compensate for this variation and produce an index that is linked to the thickness, but is virtually independent of lift-off. This index, termed as the compensated peak frequency, can be obtained from the measured multifrequency inductance spectral data using the algorithm we developed in this paper. This method has been derived through mathematical manipulation and verified by both the simulation and experimental data. Accuracy in the thickness measurements at different lift-offs proved to be within $2 \%$.
\end{abstract}

Index Terms - Eddy current testing, lift-off variation, new compensation algorithm, thickness measurement.

\section{INTRODUCTION}

$\mathbf{T}$ HE thickness of metallic plates can be measured using both multifrequency and pulse eddy current testing methods [1]-[11]. However, both the methods suffer from errors caused by the so-called lift-off effect. To address this issue, researchers have investigated a range of methods such as using different signal processing, feature extraction [12], [13], sensor structure [14]-[16], and detection principles [17]-[20]. Multifrequency eddy current sensing in the context of nondestructive testing applications has been the focus of the authors' research in recent years. Conductivity and permeability depth profiling [21], [22] and noncontact microstructure monitoring [23]-[29] have been explored.

In a recent development [30], we proposed a novel design of an eddy current sensor, composed of three coils and operating as an axial gradiometer interrogated with a multifrequency waveform. The difference in the peak frequencies of the impedance/inductance spectra from the gradiometer was used for thickness evaluation and showed to be virtually immune to lift-off variations.

Manuscript received April 12, 2016; revised June 8, 2016; accepted July 16, 2016. Date of publication August 30, 2016; date of current version November 8, 2016. This work was supported by the U.K. Engineering and Physical Sciences Research Council. The Associate Editor coordinating the review process was Dr. Sasan Bakhtiari. (Corresponding author: Wuliang Yin.)

M. Lu, A. J. Peyton, and W. Yin are with the School of Electrical and Electronic Engineering, The University of Manchester, Manchester, M60 1QD, U.K. (e-mail: wuliang.yin@manchester.ac.uk).

L. Yin is with Kunming Science and Technology University, Kunming 650051, China.

Color versions of one or more of the figures in this paper are available online at http://ieeexplore.ieee.org.

Digital Object Identifier 10.1109/TIM.2016.2600918

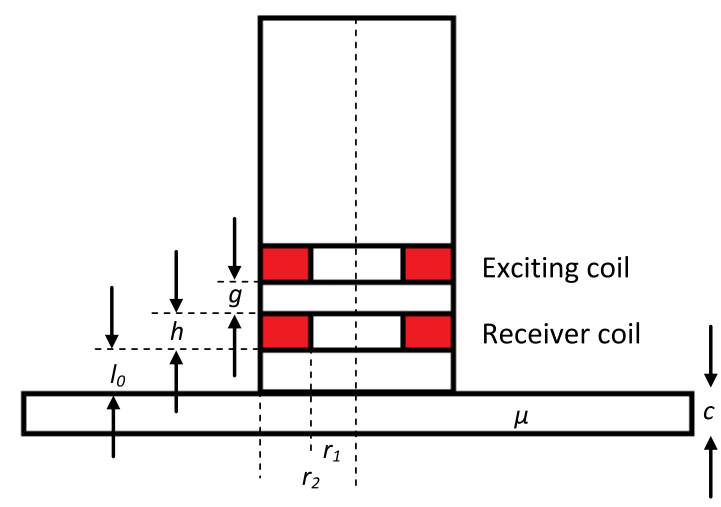

Fig. 1. Sensor configuration.

In this paper, we consider a simpler structure consisting of just one coil pair. This method has the advantages of a less complicated mechanical configuration as well as improved accuracy, and avoids the need for a precise magnetic balance, as in the case of the gradiometer. The new technique exploits two facts. First, the peak frequency of the inductance spectral signal decreases with increased lift-off, and second, the magnitude of the signal decreases with increased lift-off. An algorithm has been proposed to compensate for the change in the peak frequency. Theoretically, simulation and experiments show that the compensated peak frequency is nearly lift-off independent and therefore provide accurate thickness estimation.

\section{SENSOR DESCRIPTION}

The sensor is composed of two coils, one as excitation and the other as receiver, both of which have the same dimensions and are arranged coaxially. A schematic plot of the sensor is shown in Fig. 1, with its dimensions in Table I.

The connection of this sensor to an impedance analyzer (SL 1260) is shown in Fig. 2.

\section{TheORETICAL DERIVATION OF THE COMPEnSATEd PEAK FREQUENCy}

Previously, we have proved that the peak frequency decreases with increased lift-off [30]. For completeness, the main steps are summarized in this paper. It is also common knowledge that the signal amplitude also decreases with 
TABLE I

COIL PARAMETERS

\begin{tabular}{|c|c|}
\hline$r_{l}$ & $11.8 \mathrm{~mm}$ \\
\hline$r_{2}$ & $12 \mathrm{~mm}$ \\
\hline$l o$ (lift-off) & $0.5 \mathrm{~mm}$ \\
\hline$h$ (height) & $3 \mathrm{~mm}$ \\
\hline$g$ (gap) & $1 \mathrm{~mm}$ \\
\hline Number of turns $\mathrm{N} 1=\mathrm{N} 2$ & 20 \\
\hline
\end{tabular}

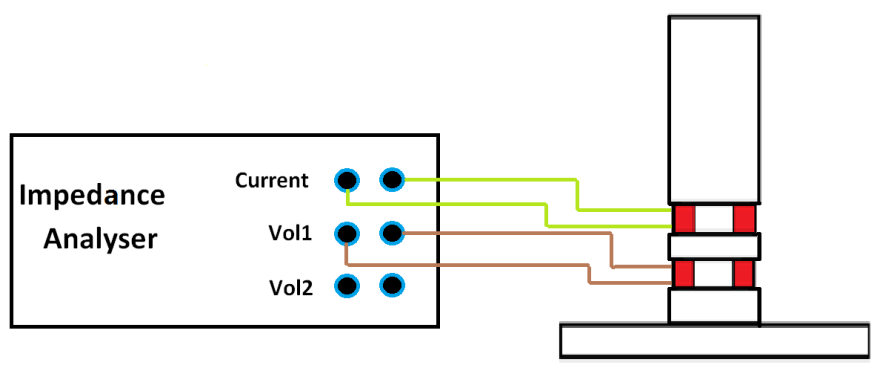

Fig. 2. Experimental wiring schematic design.

increased lift-off. Therefore, we hypothesize that an algorithm can be developed to compensate for the variation in the peak frequency with the signal amplitude. In the following, we will derive such an algorithm.

We start with the Dodd and Deeds analytical solution [31], which describes the inductance change in an air-core coil caused by a layer of nonmagnetic, metallic plates. Other similar formulas exist [32]. The difference in the complex inductance is $\Delta L(\omega)=L(\omega)-L_{A}(\omega)$, where the coil inductance above a plate is $L(\omega)$ and $L_{A}(\omega)$ is the inductance in free space.

The formulas of Dodd and Deeds [31] are

$$
\Delta L(\omega)=K \int_{0}^{\infty} \frac{P^{2}(\alpha)}{\alpha^{6}} A(\alpha) \phi(\alpha) d \alpha
$$

where

$$
\begin{aligned}
\phi(\alpha) & =\frac{\left(\alpha_{1}+\alpha\right)\left(\alpha_{1}-\alpha\right)-\left(\alpha_{1}+\alpha\right)\left(\alpha_{1}-\alpha\right) e^{2 \alpha_{1} c}}{-\left(\alpha_{1}-\alpha\right)\left(\alpha_{1}-\alpha\right)+\left(\alpha_{1}+\alpha\right)\left(\alpha_{1}+\alpha\right) e^{2 \alpha_{1} c}} \\
\alpha_{1} & =\sqrt{\alpha^{2}+j \omega \sigma \mu_{0}} \\
K & =\frac{\pi \mu_{0} N^{2}}{h^{2}\left(r_{1}-r_{2}\right)^{2}} \\
P(\alpha) & =\int_{\alpha r_{1}}^{\alpha r_{2}} x J_{1}(x) d x \\
A(\alpha) & =e^{-\alpha\left(2 l_{0}+h+g\right)}\left(e^{-2 \alpha h}+1\right)
\end{aligned}
$$

where $\mu_{0}$ denotes the permeability of free space; $N$ denotes the number of turns in the coil; $r_{1}$ and $r_{2}$ denote the inner

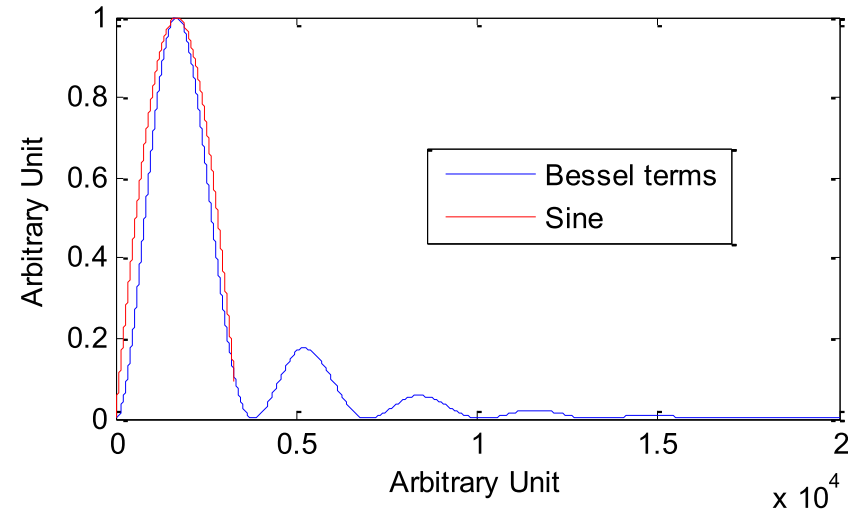

Fig. 3. Approximation of the Bessel term with a sinusoid.

and outer radii of the coil; $l_{0}$ and $h$ denote the lift-off and the height of the coil; and $c$ denotes the thickness of the plate.

Equations (1)-(6) can be approximated based on the fact that $\phi(\alpha)$ varies slowly with $\alpha$ compared with the rest of the integrand, which reaches its maximum at a characteristic spatial frequency $\alpha_{0}$ (Fig. 3). The approximation is to evaluate $\phi(\alpha)$ at $\alpha_{0}$ and take it outside of the integral

$$
\Delta L(\omega)=\phi\left(\alpha_{0}\right) \Delta L_{0}
$$

where

$$
\begin{aligned}
& \phi\left(\alpha_{0}\right) \\
& \quad=\frac{\left(\alpha_{1}+\mu \alpha_{0}\right)\left(\alpha_{1}-\mu \alpha_{0}\right)-\left(\alpha_{1}+\mu \alpha_{0}\right)\left(\alpha_{1}-\mu \alpha_{0}\right) e^{2 \alpha_{1} c}}{-\left(\alpha_{1}-\mu \alpha_{0}\right)\left(\alpha_{1}-\mu \alpha_{0}\right)+\left(\alpha_{1}+\mu \alpha_{0}\right)\left(\alpha_{1}+\mu \alpha_{0}\right) e^{2 \alpha_{1} c}} \\
& \Delta L_{0}=K \int \frac{P^{2}(\alpha)}{\alpha^{6}} A(\alpha) d \alpha .
\end{aligned}
$$

Note that in (7), the sensor phase signature is solely determined by $\phi\left(\alpha_{0}\right)$, which includes conductivity, the thickness of the conducting plate, and $\alpha_{0} . \Delta L_{0}$ is the overall magnitude of the signal, which is strongly dependent on the coil geometrical parameters but independent of the thickness and electromagnetic properties of the plate.

Substituting $e^{2 \alpha_{1} c}$ with $1+2 \alpha_{1} c$, and considering (3), (8) becomes

$$
\phi\left(\alpha_{0}\right) \approx \frac{j \omega \sigma \mu_{0} c}{j \omega \sigma \mu_{0} c+2 \alpha_{0}^{2} c+2 \alpha_{0}+2 \alpha_{0} \alpha_{1} c} .
$$

Assigning

$$
\omega_{1}=\frac{2 \alpha_{0}^{2} c+2 \alpha_{0}}{\sigma \mu_{0} c} .
$$

Equation (10) can be expressed as

$$
\phi(\alpha)=\frac{j \omega / \omega_{1}}{j \omega / \omega_{1}+1+2 \alpha_{0} \alpha_{1} c /\left(2 \alpha_{0}^{2} c+2 \alpha_{0}\right)} .
$$

In (12), it can be seen that the peak frequency for the firstorder system is approximately $\omega_{1}$ and from (11) it is concluded that the peak frequency increases with $\alpha_{0}$.

Suppose a lift-off variation of $l_{0}$ is introduced, from (6), we can see that an increase of $l_{0}$ in lift-off is equivalent to multiplying a factor $e^{-2 \alpha l_{0}}$

$$
A(\alpha)=e^{-\alpha\left(2 l_{0}+h+g\right)}\left(e^{-2 \alpha h}+1\right) .
$$


Due to the fact that $\Delta L_{0}=K \int\left(\left(P^{2}(\alpha)\right) /\left(\alpha^{6}\right)\right) A(\alpha) d \alpha$ peaks at $\alpha_{0}$ and that the squared Bessel term $P^{2}(\alpha)$ is the main contributor, a simple function $\sin ^{2}\left((\alpha \pi) /\left(2 \alpha_{0}\right)\right)$ with its maximum at $\alpha_{0}$ is used to approximate $\Delta L_{0}$

$$
\operatorname{Im}\left(\Delta L_{0}\right) \approx \operatorname{Im}\left(\Delta L_{m}\right) e^{-2 \alpha l_{0}} \sin ^{2}\left(\frac{\alpha \pi}{2 \alpha_{0}}\right)
$$

where $\Delta L_{m}$ denotes the magnitude of the inductance change when the lift-off is zero.

This simplification is applied to obtain an analytical solution for $\alpha_{0}$.

The shift in $\alpha_{0}$ due to the effect of lift-off can be predicted as follows.

The new $\alpha$ should maximize $e^{-2 \alpha l_{0}} \sin ^{2}\left(\alpha \pi / 2 \alpha_{0}\right)$ and therefore $e^{-\alpha l_{0}} \sin \left(\alpha \pi / 2 \alpha_{0}\right)$.

The maximum can be obtained by finding the stationary point for $e^{-\alpha l_{0}} \sin \left(\alpha \pi / 2 \alpha_{0}\right)$.

Let

$$
\begin{aligned}
\left(e^{-\alpha l_{0}} \sin \left(\frac{\alpha \pi}{2 \alpha_{0}}\right)\right)^{\prime}= & -l_{0} \cdot e^{-\alpha l_{0}} \sin \left(\frac{\alpha \pi}{2 \alpha_{0}}\right) \\
& +\frac{\pi}{2 \alpha_{0}} e^{-\alpha l_{0}} \cos \left(\frac{\alpha \pi}{2 \alpha_{0}}\right)=0 .
\end{aligned}
$$

And through some mathematical manipulations, a new equation can be obtained

$$
\frac{\alpha \pi}{2 \alpha_{0}}=\tan ^{-1}\left(\frac{\pi}{2 \alpha_{0} l_{0}}\right) .
$$

With a small lift-off variation, $\alpha_{0} l_{0} \ll 1$ holds and the right side can be approximated as $(\pi / 2)-\left(\left(2 \alpha_{0} l_{0}\right) / \pi\right)$.

Therefore, the revised $\alpha_{0}, \alpha_{0 r}$ is

$$
\alpha_{0 r}=\alpha_{0}-\frac{4 \alpha_{0}^{2} l_{0}}{\pi^{2}}
$$

Combining (11) with (15), $\omega_{1}$ becomes

$\omega_{1} \approx \frac{2\left(\alpha_{0}^{2} \pi^{4}-8 \pi^{2} \alpha_{0}^{3} l_{0}+16 \alpha_{0}^{4} l_{0}^{2}\right) c+2\left(\alpha_{0} \pi^{4}-\pi^{2} 4 \alpha_{0}^{2} l_{0}\right)}{\pi^{4} \sigma \mu_{0} c}$.

Combining (14) with (15), $\operatorname{Im}\left(\Delta L_{0}\right)$ becomes

$$
\begin{aligned}
\operatorname{Im}\left(\Delta L_{0}\right) & =\operatorname{Im}\left(\Delta L_{m}\right) e^{-2\left(\alpha_{0}-\frac{4 \alpha_{0}^{2} l_{0}}{\pi^{2}}\right) l_{0}} \cos ^{2}\left(\frac{2 \alpha_{0} l_{0}}{\pi}\right) \\
& =\operatorname{Im}\left(\Delta L_{m}\right) e^{-2\left(\alpha_{0}-\frac{4 \alpha_{0}^{2} l_{0}}{\pi^{2}}\right) l_{0}}\left(\frac{\cos \left(\frac{4 \alpha_{0} l_{0}}{\pi}\right)+1}{2}\right) .
\end{aligned}
$$

Considering $\alpha_{0} l_{0} \ll 1$ and based on small-angle approximation $\cos (\theta) \approx 1-\left(\left(\theta^{2}\right) / 2\right), \cos \left(\left(4 \alpha_{0} l_{0}\right) / \pi\right)$ is substituted with $1-\left(\left(\left(\left(\left(4 \alpha_{0} l_{0}\right) / \pi\right)\right)^{2}\right) / 2\right)$.
$\operatorname{Im}\left(\Delta L_{0}\right)$ becomes

$$
\operatorname{Im}\left(\Delta L_{0}\right)=\operatorname{Im}\left(\Delta L_{m}\right) e^{-2\left(\alpha_{0}-\frac{4 \alpha_{0}^{2} l_{0}}{\pi^{2}}\right) l_{0}}\left(1-\frac{4 \alpha_{0}^{2} l_{0}^{2}}{\pi^{2}}\right) .
$$

Substituting $\left(1-\left(\left(4 \alpha_{0}^{2} l_{0}^{2}\right) / \pi^{2}\right)\right)$ with $e^{-\left(\left(4 \alpha_{0}^{2} l_{0}^{2}\right) / \pi^{2}\right)}$

$$
\begin{aligned}
\operatorname{Im}\left(\Delta L_{0}\right) & =\operatorname{Im}\left(\Delta L_{m}\right) e^{-2\left(\alpha_{0}-\frac{4 \alpha_{0}^{2} l_{0}}{\pi^{2}}\right) l_{0}} e^{-\frac{4 \alpha_{0}^{2} l_{0}^{2}}{\pi^{2}}} \\
& =\operatorname{Im}\left(\Delta L_{m}\right) e^{-2\left(\alpha_{0}-\frac{2 \alpha_{0}^{2} l_{0}}{\pi^{2}}\right) l_{0}} .
\end{aligned}
$$

Taking the natural logarithmic operation of both the sides, we arrive at

$$
\ln \frac{\operatorname{Im}\left(\Delta L_{0}\right)}{\operatorname{Im}\left(\Delta L_{m}\right)}=-2\left(\alpha_{0}-\frac{2 \alpha_{0}^{2} l_{0}}{\pi^{2}}\right) l_{0} .
$$

And further

$$
4 \alpha_{0}^{2} l_{0}^{2}-2 \pi^{2} \alpha_{0} l_{0}-\pi^{2} \ln \frac{\operatorname{Im}\left(\Delta L_{0}\right)}{\operatorname{Im}\left(\Delta L_{m}\right)}=0 .
$$

This is now a quadratic equation with $\alpha_{0} l_{0}$ as its variable.

Therefore, the solution for $\alpha_{0} l_{0}$ is

$$
\alpha_{0} l_{0}=\frac{\pi^{2}-\sqrt{\pi^{4}+4 \pi^{2} \ln \frac{\operatorname{Im}\left(\Delta L_{0}\right)}{\operatorname{Im}\left(\Delta L_{m}\right)}}}{4} .
$$

The other solution

$$
\alpha_{0} l_{0}=\frac{\pi^{2}+\sqrt{\pi^{4}+4 \pi^{2} \ln \frac{\operatorname{Im}\left(\Delta L_{0}\right)}{\operatorname{Im}\left(\Delta L_{m}\right)}}}{4}
$$

does not satisfy the small lift-off condition $\alpha_{0} l_{0} \ll 1$ and therefore is discarded.

From (19), lift-off can be estimated as

$$
l_{0}=\frac{\pi^{2}-\sqrt{\pi^{4}+4 \pi^{2} \ln \frac{\operatorname{Im}\left(\Delta L_{0}\right)}{\operatorname{Im}\left(\Delta L_{m}\right)}}}{4 \alpha_{0}} .
$$

Combining (16) with (20), the peak frequency with a lift-off of $l_{0}$ becomes

$\omega_{1}=\frac{2 \alpha_{0}^{2} C\left(\pi^{2}+4 \ln \frac{\operatorname{Im}\left(\Delta L_{0}\right)}{\operatorname{Im}\left(\Delta L_{m}\right)}\right)+2 \alpha_{0} \pi \sqrt{\pi^{2}+4 \ln \frac{\operatorname{Im}\left(\Delta L_{0}\right)}{\operatorname{Im}\left(\Delta L_{m}\right)}}}{\pi^{2} \sigma \mu_{0} c}$.

Equation (21) becomes a quadratic equation with an unknown $\alpha_{0}$

$$
\begin{aligned}
& 2 c\left(\pi^{2}+4 \ln \frac{\operatorname{Im}\left(\Delta L_{0}\right)}{\operatorname{Im}\left(\Delta L_{m}\right)}\right) \alpha_{0}^{2} \\
& \quad+2 \pi \sqrt{\pi^{2}+4 \ln \frac{\operatorname{Im}\left(\Delta L_{0}\right)}{\operatorname{Im}\left(\Delta L_{m}\right)}} \alpha_{0}-\omega_{1} \pi^{2} \sigma \mu_{0} c=0 .
\end{aligned}
$$

And the solution is (22), as shown at the bottom of this page.

$$
\begin{aligned}
\alpha_{0} & =\frac{-\pi \sqrt{\pi^{2}+4 \ln \frac{\operatorname{Im}\left(\Delta L_{0}\right)}{\operatorname{Im}\left(\Delta L_{m}\right)}}+\sqrt{\pi^{2}\left(2 \omega_{1} \sigma \mu_{0} c^{2}+1\right)\left(\pi^{2}+4 \ln \frac{\operatorname{Im}\left(\Delta L_{0}\right)}{\operatorname{Im}\left(\Delta L_{m}\right)}\right)}}{2 c\left(\pi^{2}+4 \ln \frac{\operatorname{Im}\left(\Delta L_{0}\right)}{\operatorname{Im}\left(\Delta L_{m}\right)}\right)} \\
& =\frac{\pi\left(\sqrt{\left(1+2 \omega_{1} \sigma \mu_{0} c^{2}\right)}-1\right)}{2 c \sqrt{\pi^{2}+4 \ln \frac{\operatorname{Im}\left(\Delta L_{0}\right)}{\operatorname{Im}\left(\Delta L_{m}\right)}}}
\end{aligned}
$$




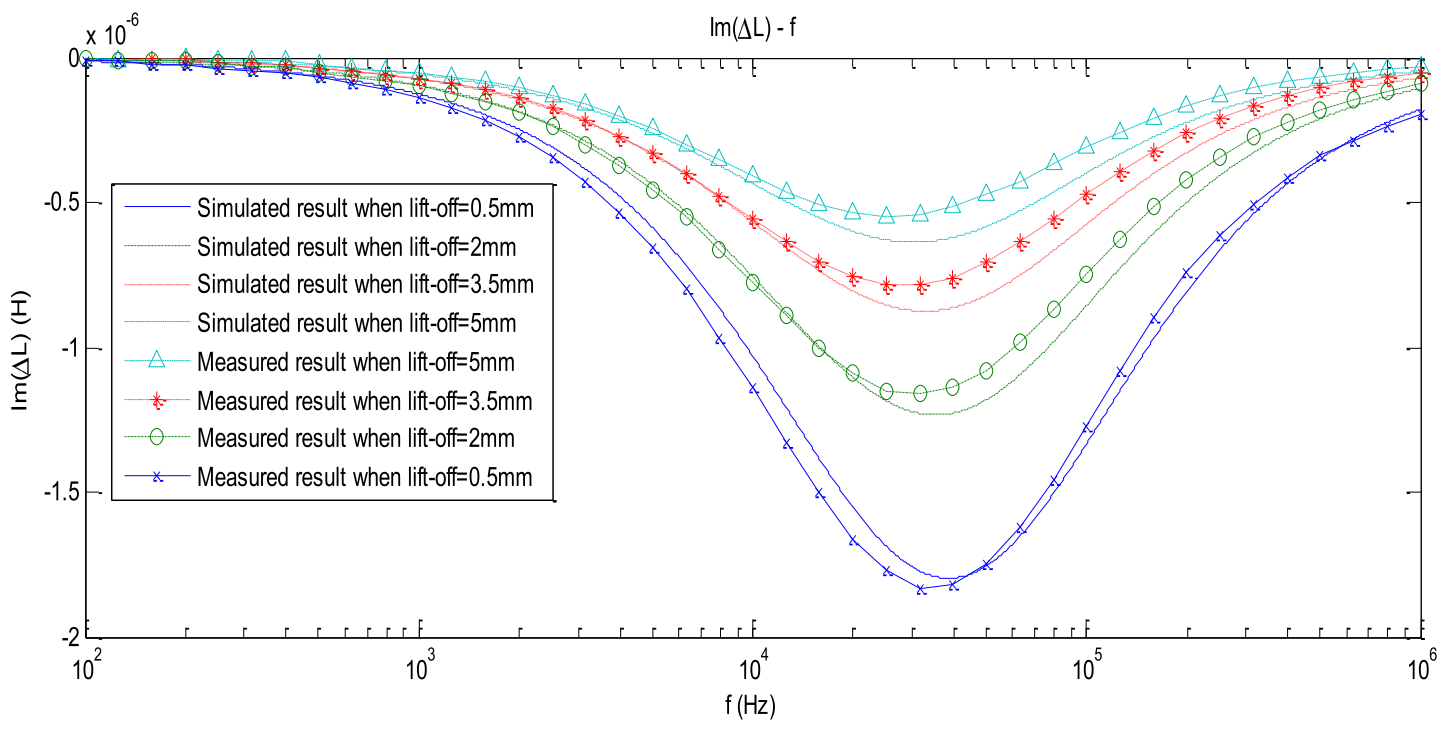

Fig. 4. Simulation and experimental results of imaginary parts of $\Delta L$ for a $22-\mu \mathrm{m}$ aluminum plate at a range of lift-offs.

Therefore, by combining (11) with (22), the original peak frequency (peak frequency prior to introducing the lift-off $l_{0}$ ) can be obtained as in (23), shown at the bottom of this page.

It can be seen in (23) that through a compensation scheme, and using the knowledge of the peak frequency and the amplitude at a certain lift-off, the original peak frequency (peak frequency prior to introducing the lift-off $l_{0}$ ) can be recovered.

Further approximation can be carried out by considering $\alpha_{0} c \ll 1$. The peak frequency of the imaginary part of the inductance in (21) becomes

$$
\omega_{1} \approx \frac{2 \alpha_{0} \sqrt{\pi^{2}+4 \ln \frac{\operatorname{Im}\left(\Delta L_{0}\right)}{\operatorname{Im}\left(\Delta L_{m}\right)}}}{\pi \sigma \mu_{0} c} .
$$

Equation (22) becomes

$$
\alpha_{0} \approx \frac{\pi \sigma \mu_{0} \omega_{1} c}{2 \sqrt{\pi^{2}+4 \ln \frac{\operatorname{Im}\left(\Delta L_{0}\right)}{\operatorname{Im}\left(\Delta L_{m}\right)}}} .
$$

Therefore, the compensated peak frequency (peak frequency prior to introducing the lift-off $l_{0}$ ) becomes

$$
\omega_{0}^{\prime} \approx \frac{2 \alpha_{0}}{\sigma \mu_{0} c}=\frac{\pi \omega_{1}}{\sqrt{\pi^{2}+4 \ln \frac{\operatorname{Im}\left(\Delta L_{0}\right)}{\operatorname{lm}\left(\Delta L_{m}\right)}}} .
$$

So, the thickness reduces to

$$
c=\frac{2 \alpha_{0}}{\sigma \mu_{0} \omega_{0}^{\prime}}=\frac{2 \alpha_{0} \sqrt{\pi^{2}+4 \ln \frac{\operatorname{Im}\left(\Delta L_{0}\right)}{\operatorname{Im}\left(\Delta L_{m}\right)}}}{\pi \sigma \mu_{0} \omega_{1}} .
$$

Therefore from (27) it can be seen that as lift-off increases, the measured peak frequency decreases, but the numerator term also decreases to compensate for this, so that the compensated peak frequency and accurate thickness can be recovered.

\section{Simulations AND EXPERIMENTS}

Experiments and simulations were carried out to verify the performance of the compensation algorithm; the compensated peak frequency and the compensated thickness measurements at different lift-offs were compared. Here, the imaginary part of the inductance is defined from the mutual impedance of the coils

$$
\begin{aligned}
\operatorname{Im}(\Delta L) & =\operatorname{Im}\left(\frac{Z(f)-Z_{\mathrm{air}}(f)}{j 2 \pi f}\right) \\
& =\operatorname{Re}\left(\frac{-\left(Z(f)-Z_{\mathrm{air}}(f)\right)}{2 \pi f}\right)
\end{aligned}
$$

where $Z(f)$ denotes the impedance of the coil in the presence of a metallic plate, while $Z_{\text {air }}(f)$ is that of the coil in air.

\section{A. Simulations}

The simulated sensor configuration is shown in Table I. The simulated targets are aluminum plates with a conductivity of $38.2 \mathrm{MS} / \mathrm{m}$ and thickness of 22 and $44 \mu \mathrm{m}$ under varying lift-offs $0.5,2,3.5$, and $5 \mathrm{~mm}$. The simulations were realized by a custom developed solver using MATLAB. The solver can be used to calculate the Dodd and Deeds solution [31] (1)-(6)

$$
\begin{aligned}
\omega_{0} & =\frac{2 \alpha_{0}^{2} c+2 \alpha_{0}}{\sigma \mu_{0} c} \\
& =\frac{\pi^{2}\left(\sqrt{\left(1+2 \omega_{1} \sigma \mu_{0} c^{2}\right)}-1\right)^{2}+2 \pi\left(\sqrt{\left(1+2 \omega_{1} \sigma \mu_{0} c^{2}\right)}-1\right) \sqrt{\pi^{2}+4 \ln \frac{\operatorname{Im}\left(\Delta L_{0}\right)}{\operatorname{Im}\left(\Delta L_{m}\right)}}}{2 \sigma \mu_{0} c^{2}\left(\pi^{2}+4 \ln \frac{\operatorname{Im}\left(\Delta L_{0}\right)}{\operatorname{Im}\left(\Delta L_{m}\right)}\right)}
\end{aligned}
$$


TABLE II

ThickNess MEASUREMENTS FOR DifFERENT THICKNESSES AND LIFT-OFFS

\begin{tabular}{|c|c|c|c|c|}
\hline $\begin{array}{c}\text { Lift-off } \\
(\mathrm{mm})\end{array}$ & $\begin{array}{c}\text { Actual } \\
\text { thickness } \\
(\mu \mathrm{m})\end{array}$ & $\begin{array}{c}\text { Thickness calculated } \\
\text { from original as } \\
\text { measured peak } \\
\text { frequency }(\mu \mathrm{m})\end{array}$ & $\begin{array}{c}\text { Thickness calculated } \\
\text { from Compensated peak } \\
\text { frequency by the simple } \\
2 \text { coils probe }(\mu \mathrm{m})\end{array}$ & $\begin{array}{c}\text { Thickness tested } \\
\text { from previous } \\
\text { Triple-Coil probe } \\
(\mu \mathrm{m})\end{array}$ \\
\hline 1.5 & 22 & 23.1 & 22.2 & 22.14 \\
\hline 1.5 & 44 & 46.3 & 44.3 & 43.21 \\
\hline 2 & 22 & 23.5 & 22.3 & 21.42 \\
\hline 2 & 44 & 47 & 44.2 & 44.83 \\
\hline 3.5 & 22 & 23.8 & 22.2 & 4.22 \\
\hline 3.5 & 44 & 47.9 & 44.4 & 44.95 \\
\hline
\end{tabular}

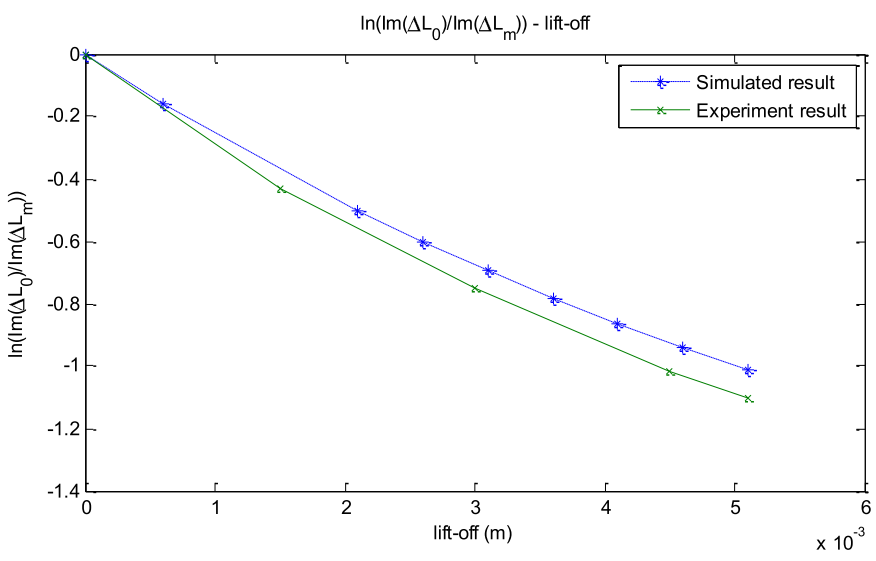

Fig. 5. Simulation and experimental results. Natural logarithmic of $\operatorname{Im}(\Delta L)$ ratio for the $22-\mu \mathrm{m}$ sample at a range of lift-offs.

and calculate the thickness and peak frequency using (24)-(27). The solver can take a range of different parameters such as frequency, sample conductivity, thickness, and lift-off. In addition, the solver have been converted and packaged into an executable program.

\section{B. Experimental Setup}

The sensor configuration and the test pieces are the same as that of the simulations. And the multifrequency response of the sensor was obtained by an SL 1260 impedance analyzer with frequency sweeping mode.

It can be seen from Fig. 4 that the peak frequency decreases as lift-off increases and, at the same time, the magnitude of the signal decreases with increased lift-offs. Fig. 5 shows the experimental result.

As can be seen from Fig. 6, the compensated peak frequency decreases following initial lift-off but remains almost constant for larger lift-offs, i.e., peak frequency is virtually immune to lift-off variations for larger lift-offs. Tests were carried out to verify this method and the thickness measurement results are shown in Table II for varying thicknesses and lift-offs. Here, the small thickness is defined as $c \ll 1 / \alpha_{0}$, so it depends on the size of the coil. In our case, this value is generally $<1 \mathrm{~mm}$. It can be seen from Fig. 6 that both the compensated and the uncompensated peak frequency are smaller than the actual

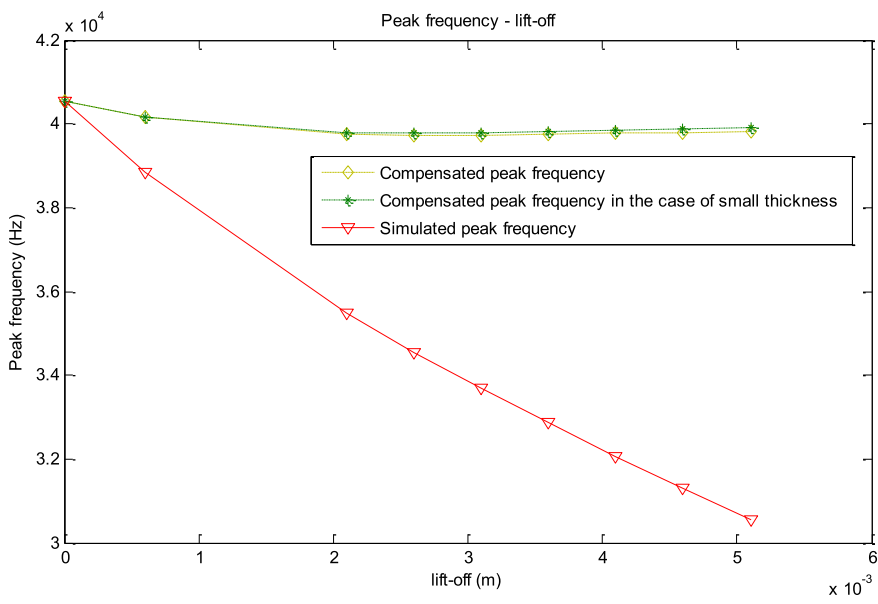

(a)

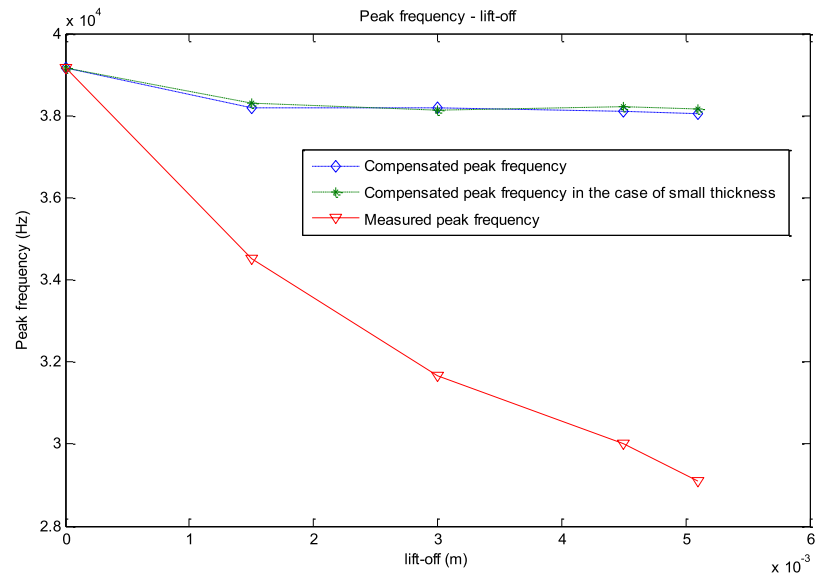

(b)

Fig. 6. Comparisons of as-measured (uncompensated) and compensated peak frequencies for the $22-\mu \mathrm{m}$ aluminum plate at a range of lift-offs. (a) Simulation results. (b) Experimental results.

peak frequency. Since the thickness is inversely proportional to the peak frequency [see (27)], therefore, the calculated thickness is larger than the actual thickness.

In addition, a comparison of the thickness tested from the previous Triple-Coil probe is also added in Table II. It can be seen that the method reported in this paper has in general improved accuracy in thickness measurement and the coil has a simpler structure. 


\section{CONClusion}

This paper has considered a compensation scheme for reducing the errors in multifrequency eddy current thickness measurements of metallic plates. The peak frequency decreases as lift-offs increase and an algorithm has been developed that can compensate for this variation frequency and produce an index that is linked to thickness but is virtually independent of lift-off. Both the simulation and experimental results show that the compensated peak frequency is almost immune to the lift-off variations. This is an important feature as a lift-off variation is unavoidable in many practical applications. Although the algorithm involved is slightly more complicated, this new approach has the advantages of a less complicated mechanical configuration as well as improved accuracy, and avoids the need for a precise magnetic balance, as in the case of the three-coil configuration [30].

An SL 1260 impedance analyzer working in a swept frequency mode was used to acquire the multifrequency data in this paper. However, multifrequency impedances can also be abstracted simultaneously using composite multisine waveform excitation followed by fast Fourier transform operations as in [33].

\section{REFERENCES}

[1] J. C. Moulder, E. Uzal, and J. H. Rose, "Thickness and conductivity of metallic layers from eddy current measurements," Rev. Sci. Instrum. vol. 63, no. 6, pp. 3455-3465, 1992.

[2] Y. Nonaka, "A double coil method for simultaneously measuring the resistivity, permeability, and thickness of a moving metal sheet," IEEE Trans. Instrum. Meas., vol. 45, no. 2, pp. 478-482, Apr. 1996.

[3] A. Sethuraman and J. H. Rose, "Rapid inversion of eddy current data for conductivity and thickness of metal coatings," J. Nondestruct. Eval., vol. 14, no. 1, pp. 39-46, 1995.

[4] Z. Qu, Q. Zhao, and Y. Meng, "Improvement of sensitivity of eddy current sensors for nano-scale thickness measurement of $\mathrm{Cu}$ films," NDT E Int., vol. 61, pp. 53-57, Jan. 2014.

[5] W. Yin, A. J. Peyton, and S. J. Dickinson, "Simultaneous measurement of distance and thickness of a thin metal plate with an electromagnetic sensor using a simplified model," IEEE Trans. Instrum. Meas., vol. 53, no. 4, pp. 1335-1338, Aug. 2004.

[6] W. Yin and A. J. Peyton, "Thickness measurement of metallic plates with an electromagnetic sensor using phase signature analysis," IEEE Trans. Instrum. Meas., vol. 57, no. 8, pp. 1803-1807, Aug. 2008.

[7] W. Yin and A. J. Peyton, "Thickness measurement of non-magnetic plates using multi-frequency eddy current sensors," NDT E Int., vol. 40, no. 1, pp. 43-48, Jan. 2007.

[8] E. Pinotti and E. Puppin, "Simple lock-in technique for thickness measurement of metallic plates," IEEE Trans. Instrum. Meas., vol. 63, no. 2, pp. 479-484, Feb. 2014.

[9] C.-C. Tai, J. H. Rose, and J. C. Moulder, "Thickness and conductivity of metallic layers from pulsed eddy-current measurements," Rev. Sci. Instrum., vol. 67, no. 11, pp. 3965-3972, 1996.

[10] D. Vasić, V. Bilas, and B. Šnajder, "Analytical modelling in lowfrequency electromagnetic measurements of steel casing properties," NDT E Int., vol. 40, no. 2, pp. 103-111, 2007.

[11] H.-C. Yang and C.-C. Tai, "Pulsed eddy-current measurement of a conducting coating on a magnetic metal plate," Meas. Sci. Technol., vol. 13, no. 8, pp. 1259-1265, 2002.

[12] G. Y. Tian and A. Sophian, "Reduction of lift-off effects for pulsed eddy current NDT," NDT E Int., vol. 38, no. 4, pp. 319-324, 2005.

[13] S. Giguère and S. J. M. Dubois, "Pulsed eddy current: Finding corrosion independently of transducer lift-off," In Rev. Prog. Quant. Nondestruct. Eval., vol. 19, no. 1, pp. 449-456, 2000. [Online]. Available: http://dx.doi.org/10.1063/1.1306083

[14] H. Hoshikawa and K. Koyama, "A new eddy current surface probe without lift-off noise," in Proc. 10th APCNDT, Brisbane, QLD, Australia, 2001, pp. 275-8575.
[15] A. Reza Khalaj, M. Ravan, S. H. H. Sadeghi, and R. Moini, "Using $\mathrm{AC}$ field measurement data at an arbitrary liftoff distance to size long surface-breaking cracks in ferrous metals," NDT E Int., vol. 41, no. 3, pp. 169-177, 2008 .

[16] S. Li, H. Songling, and Z. Wei, "Development of differential probes in pulsed eddy current testing for noise suppression," Sens. Actuators A, Phys., vol. 135, no. 2, pp. 675-679, 2007.

[17] O. Postolache, A. L. Ribeiro, and H. G. Ramos, "GMR array uniform eddy current probe for defect detection in conductive specimens," Measurement, vol. 46, no. 10, pp. 4369-4378, Dec. 2013.

[18] D. F. He and M. Yoshizawa, "Dual-frequency eddy-current NDE based on high- $T_{\mathrm{c}}$ RF SQUID," Phys. C, Supercond., vol. 383, no. 3, pp. 223-226, 2002.

[19] J. Kral, R. Smid, H. M. G. Ramos, and A. L. Ribeiro, "The lift-off effect in eddy currents on thickness modeling and measurement," IEEE Trans. Instrum. Meas., vol. 62, no. 7, pp. 2043-2049, Jul. 2013.

[20] A. L. Ribeiro, H. G. Ramos, and J. C. Arez, "Liftoff insensitive thickness measurement of aluminum plates using harmonic eddy current excitation and a GMR sensor," Measurement, vol. 45, no. 9, pp. 2246-2253, 2012.

[21] W. Yin, S. J. Dickinson, and A. J. Peyton, "Imaging the continuous conductivity profile within layered metal structures using inductance spectroscopy," IEEE Sensors J., vol. 5, no. 2, pp. 161-166, Apr. 2005.

[22] W. Yin, S. J. Dickinson, and A. J. Peyton, "Evaluating the permeability distribution of a layered conductor by inductance spectroscopy," IEEE Trans. Magn., vol. 42, no. 11, pp. 3645-3651, Nov. 2006.

[23] G. Betta, L. Ferrigno, M. Laracca, P. Burrascano, M. Ricci, and G. Silipigni, "An experimental comparison of multi-frequency and chirp excitations for eddy current testing on thin defects," Measurement, vol. 63, pp. 207-220, Mar. 2015.

[24] R. J. Haldane, W. Yin, M. Strangwood, A. J. Peyton, and C. L. Davis, "Multi-frequency electromagnetic sensor measurement of ferrite/austenite phase fraction-Experiment and theory," Scr. Mater. vol. 54, no. 10, pp. 1761-1765, 2006.

[25] A. Bernieri, G. Betta, L. Ferrigno, and M. Laracca, "Crack depth estimation by using a multi-frequency ECT method," IEEE Trans. Instrum. Meas., vol. 62, no. 3, pp. 544-552, Mar. 2013.

[26] W. Yin, A. J. Peyton, M. Strangwood, and C. L. Davis, "Exploring the relationship between ferrite fraction and morphology and the electromagnetic properties of steel," J. Mater. Sci., vol. 42, no. 16 , pp. 6854-6861, 2007.

[27] W. Yin, X. J. Hao, A. J. Peyton, M. Strangwood, and C. L. Davis, "Measurement of permeability and ferrite/austenite phase fraction using a multi-frequency electromagnetic sensor," NDT E Int., vol. 42, no. 1, pp. 64-68, 2009.

[28] L. S. Rosado, P. M. Ramos, and M. Piedade, "Real-time processing of multifrequency eddy currents testing signals: Design, implementation, and evaluation," IEEE Trans. Instrum. Meas., vol. 63, no. 5, pp. 1262-1271, May 2014.

[29] W. Yin, R. Binns, S. J. Dickinson, C. Davis, and A. J. Peyton, "Analysis of the liftoff effect of phase spectra for eddy current sensors," IEEE Trans. Instrum. Meas., vol. 56, no. 6, pp. 2775-2781, Dec. 2007.

[30] W. Yin and K. Xu, "A novel triple-coil electromagnetic sensor for thickness measurement immune to lift-off variations," IEEE Trans. Instrum. Meas., vol. 65, no. 1, pp. 164-169, Jan. 2016.

[31] C. V. Dodd and W. E. Deeds, "Analytical solutions to eddy-current probe-coil problems," J. Appl. Phys., vol. 39, no. 6, pp. 2829-2839, 1968.

[32] S. K. Burke and R. J. Ditchburn, "Mutual impedance of planar eddycurrent driver-pickup spiral coils," Res. Nondestruct. Eval., vol. 19, pp. 1-19, Jan. 2008.

[33] W. Yin, S. J. Dickinson, and A. J. Peyton, "A multi-frequency impedance analysing instrument for eddy current testing," Meas. Sci. Technol., vol. 17 , no. 2 , pp. $393-402,2006$

Mingyang Lu is currently pursuing the Ph.D. degree under the supervision of Dr. W. Yin with the School of Electrical and Electronic Engineering, University of Manchester, Manchester, U.K.

$\mathrm{He}$ is involved in developing a finite-element model to solve electromagnetic simulation by taking into account the random geometry and material properties (including microstructure). He is also developing software to increase efficiency of simulations to avoid remeshing, for example, to consider a moving sensor as a moving effective field above a flaw (nondestructive testing application).

Liyuan Yin, photograph and biography not available at the time of publication. 
Anthony J. Peyton received the B.Sc. degree in electrical and electronics engineering and the Ph.D. degree from the University of Manchester Institute of Science and Technology (UMIST), Manchester, U.K., in 1983 and 1986, respectively.

He was a Principal Engineer with Kratos Analytical Ltd., Manchester, in 1989, where he was involved in developing precision electronic instrumentation systems for magnetic sector and quadrupole mass spectrometers. He joined the Process Tomography Group, UMIST, where he was a Lecturer. In 1996, he was a Senior Lecturer with Lancaster University, Lancaster, U.K., where he was a Reader in Electronic Instrumentation in 2001, and a Professor in 2004. Since 2004, he has been a Professor of Electromagnetic Tomography Engineering with the University of Manchester, Manchester. His current research interests include instrumentation, applied sensor systems, and electromagnetics.
Wuliang Yin (M'05-SM'06) was appointed as an MT Sponsored Lecturer with the School of Electrical and Electronic Engineering, University of Manchester, Manchester, U.K., in 2012, and was promoted to Senior Lecturer in 2016. He has authored one book, more than 140 papers, and was granted more than ten patents in the area of electromagnetic (EM) sensing and imaging. He currently leads several major grants from U.K. government bodies including TSB and EPSRC and is involved in several European Union projects.

Dr. Yin was a recipient of the 2014 and 2015 Williams Award from the Institute of Materials, Minerals and Mining for his contribution in applying EM imaging in the steel industry. He also received the Science and Technology Award from the Chinese Ministry of Education in 2000. 